University of Wollongong

Research Online

Australian Institute for Innovative Materials -

Papers

Australian Institute for Innovative Materials

January 2014

Intrinsic reduction of the ordered $4 \mathrm{f}$ magnetic moments in semiconducting rare-earth nitride thin films: DyN, ErN, and HoN

David L. Cortie

University of Wollongong, dlc422@uowmail.edu.au

J Brown

United States Naval Academy

S Bruck

ANSTO, University of New South Wales

T Saerbeck

ANSTO

Jason P. Evans

University of New South Wales

See next page for additional authors

Follow this and additional works at: https://ro.uow.edu.au/aiimpapers

Research Online is the open access institutional repository for the University of Wollongong. For further information contact the UOW Library: research-pubs@uow.edu.au 


\section{Intrinsic reduction of the ordered $4 \mathrm{f}$ magnetic moments in semiconducting rare- earth nitride thin films: DyN, ErN, and HoN}

\section{Keywords}

reduction, earth, rare, thin, films, dyn, ern, intrinsic, magnetic, hon, 4f, nitride, ordered, semiconducting, moments

\section{Publication Details}

Cortie, D. L., Brown, J. D., Bruck, S., Saerbeck, T., Evans, J. P., Fritzsche, H., Wang, X., Downes, J. E. \& Klose, F. (2014). Intrinsic reduction of the ordered $4 \mathrm{f}$ magnetic moments in semiconducting rare-earth nitride thin films: DyN, ErN, and HoN. Physical Review B: Condensed Matter and Materials Physics, 89 (6),

064424-1-064424-7.

\section{Authors}

David L. Cortie, J Brown, S Bruck, T Saerbeck, Jason P. Evans, H Fritzsche, Xiaolin Wang, J E. Downes, and Frank Klose 


\title{
Intrinsic reduction of the ordered $4 f$ magnetic moments in semiconducting rare-earth nitride thin films: DyN, ErN, and HoN
}

\author{
D. L. Cortie, ${ }^{1,2,{ }^{*}}$ J. D. Brown, ${ }^{3}$ S. Brück, ${ }^{2,4}$ T. Saerbeck, ${ }^{2, \dagger}$ J. P. Evans, ${ }^{3}$ H. Fritzsche, ${ }^{5}$ X. L. Wang, ${ }^{1}$ \\ J. E. Downes, ${ }^{3, \ddagger}$ and F. Klose ${ }^{2}$ \\ ${ }^{1}$ The Institute for Superconducting and Electronic Materials, The University of Wollongong, Wollongong, New South Wales 2522, Australia \\ ${ }^{2}$ Australian Nuclear Science and Technology Organisation, Lucas Heights, New South Wales 2234, Australia \\ ${ }^{3}$ Department of Physics and Astronomy, Macquarie University, North Ryde, New South Wales 2109, Australia \\ ${ }^{4}$ The University of New South Wales, Sydney, New South Wales 2052, Australia \\ ${ }^{5}$ Canadian Neutron Beam Centre, Chalk River Laboratories, Building 459, Chalk River, Ontario K0J 1J0, Canada \\ (Received 16 September 2013; revised manuscript received 3 February 2014; published 26 February 2014)
}

\begin{abstract}
Polarized neutron reflectometry and x-ray reflectometry were used to determine the nanoscale magnetic and chemical depth profiles of the heavy rare-earth nitrides HoN, ErN, and DyN in the form of 15- to 40-nm-thick films. The net ferromagnetic components are much lower than the predictions of density-functional theory and Hund's rules for a simple ferromagnetic ground state in these $4 f$ ionic materials, which points to the intrinsic contribution of crystal-field effects and noncollinear spin structures. The magnetic moment per rare-earth ion was determined as a function of temperature in the range 5-100 K at fields of $1-4 \mathrm{~T}$. It is demonstrated that the films are stoichiometric within 1-3\% and magnetically homogeneous on the nanometer scale.
\end{abstract}

DOI: 10.1103/PhysRevB.89.064424

PACS number(s): 61.05.fj, 75.50.Pp

\section{INTRODUCTION}

The heavier members of the rare-earth nitride (REN) family (GdN, DyN, HoN, and ErN) were predicted to have the rare combination of large magnetic moments, intrinsic ferromagnetism, and semiconducting properties [1,2]. In the past decade, there have been many studies confirming ferromagnetism and wide-band-gap semiconducting behavior of $\mathrm{GdN}$ thin films [3-6]. On the other hand, very little experimental information is available for DyN, ErN, and HoN films $[7,8]$. In particular, the magnetic ground state in thin films of HoN has not been reported. From a crystallographic point-of-view, the rare-earth (RE) nitride and RE metals have a similar interatomic separation that is lower than those in the other monopnictides (REX, X $=\mathrm{P}, \mathrm{As}, \mathrm{Sb}, \mathrm{Bi}$ ). Figure 1 compares the crystal structure of Ho metal and HoN obtained from bulk crystallographic data $[9,10]$. The simple structure and short RE-RE distances of the REN materials lead to an array of potentially useful properties for spin electronics: halfmetallic, zero-gap, and semiconducting states were predicted to coexist with magnetic order in various ways across the series $[1,2]$. This has culminated in the recent incorporation of some of these materials into actual devices [11,12]. Past work has been hindered by the tendency of these materials to spontaneously oxidize if exposed to air. In earlier neutron and magnetometry studies, the question of stoichiometry was raised $[9,13]$, and even low oxygen doping levels $(0.05 \%)$ were found to dramatically alter the properties of $\mathrm{GdN}$ [14]. It is unknown whether DyN, ErN, and HoN suffer from the same level of sensitivity. Recently thin-film capping layers have been shown to circumvent the oxidation problem by providing a protective coating for certain REN films including $\operatorname{GdN}[3,6,7]$ with varying degrees of success [15]. While the

\footnotetext{
*d.1.cortie@gmail.com

†Now at Department of Physics, University of California San Diego, La Jolla, CA 92093, USA

†james.downes@mq.edu.au
}

magnetic and electronic structures for some materials have been reported $[5,7,16,17]$, many open questions remain, and it was only quite recently that similar ex situ experiments have been demonstrated for HoN films [17]. The absence of reports regarding the magnetic properties of $\mathrm{HoN}$ thin films is remarkable given the known semiconducting behavior of HoN [17] and the net ferromagnetic behavior found in bulk form [9]. In this work, we studied thin films of ErN, DyN, and HoN using the depth-sensitive magnetic probe of neutron reflectometry, which is a nondestructive method to study the buried chemical and magnetic depth profile.

\section{SAMPLE DEPOSITION AND CHARACTERIZATION}

DyN, HoN, and ErN films measuring 15-40 nm were deposited on $\mathrm{Al}_{2} \mathrm{O}_{3}$ (001) substrates using the low-energy ion-assisted deposition technique (IAD). The optimized deposition temperature for the DyN, HoN, and ErN films was 740,730 , and $720^{\circ} \mathrm{C}$, respectively, and the pressure was 0.1 $\mathrm{Pa} \mathrm{N}_{2}$. The current of the nitrogen ions for each of the three films was 150,90 , and $160 \mu \mathrm{A} / \mathrm{cm}^{2}$, respectively, which was found to lead to the best crystal quality for the nitride. The films were passivated with a 25-nm yttria-stabilized zirconia (YSZ) layer deposited using rf magnetron sputtering at ambient temperature. X-ray reflectometry and diffraction were conducted on a Panalytical X'Pert Pro laboratory source using $\mathrm{Cu}-\mathrm{K}_{\alpha}$ radiation $(\lambda=0.154 \mathrm{~nm})$. A 5-T Quantum Design magnetic property measurement system (MPMS) was used for magnetic characterization. Polarized neutron reflectometry was performed at the D3 reflectometer and PLATYPUS reflectometer [18]. D3 is located at the National Research Universal (NRU) reactor in Chalk River, Canada and operates at a wavelength of $0.237 \mathrm{~nm}$ using a pyrolytic graphite (PG) monochromator along with a PG filter to reduce the higher-order contributions of the monochromator. To facilitate high-field studies, the polarization setup on D3 consists of a cryomagnet in an asymmetric configuration [19]. PLATYPUS is a time-of-flight reflectometer located at the OPAL reactor in Sydney, Australia, maintaining a polarized beam in a 


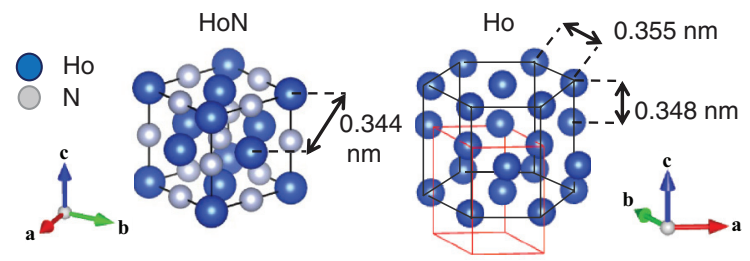

FIG. 1. (Color online) (a) A comparison of the crystal structure of the face-centered cubic rare-earth nitride (left) with the hexagonal rare-earth metal (right). Dotted lines indicate neighbor lengths. Lighter lines (red) indicate the primitive unit cell for metallic Ho.

0.25- to 1.3-nm-wavelength spectrum [20]. Polarized neutron reflectometry measures the reflected neutron intensity near a material's critical edge of total reflection [21]. In such a measurement, the independent variables are the wavelength and spin state of the incoming neutron, the angle of incidence, the applied field, and the temperature. The intensity of the reflected neutron beam in different spin states is measured as a function of reflected angle and wavelength, usually expressed in terms of the scattering vector:

$$
Q=\frac{4 \pi \sin (\theta)}{\lambda} .
$$

In this work, the results presented refer to the case of specular reflection where the incident and reflected angle were equal, since there was no indication of off-specular scattering. The incoming neutron was quantized to one of the two possible spin states (notated here as + and - ), and the outgoing neutron was analyzed to determine if neutron spin-flip scattering had occurred. The reflected intensities for each of the four possibilities were notated as $\mathrm{R}_{++}, \mathrm{R}_{--}, \mathrm{R}_{+-}$, and $\mathrm{R}_{-+}$, where the first subscript is the spin state of the incoming neutron and the second refers to the spin state of the outgoing neutron. Film magnetization in the direction of the applied field causes splitting between the $\mathrm{R}_{++}$and $\mathrm{R}_{--}$channel, known as spin asymmetry, whereas spin-flip intensity $\mathrm{R}_{+-}=\mathrm{R}_{-+}$only occurs for magnetization that lies in the film plane and is noncollinear with both the applied field and the neutron polarization. X-ray and neutron reflected intensities were fitted by using the Motofit [22] and SimulRefl [23] software packages to provide a precise determination of the chemical and magnetic profile of the films.

\section{RESULTS}

Under the deposition conditions described, the lattice constants of typical DyN, HoN, and ErN films were found by $\mathrm{X}$-ray diffraction to be $4.895,4.871$, and $4.825 \AA$, respectively, and the films were found to be polycrystalline with a random grain orientation. Figure 2 shows the x-ray reflectometry patterns for the DyN, HoN, and ErN thin films obtained at $300 \mathrm{~K}$, along with the best fits to the data using the scattering length density (SLD) profile shown in the inset. The $\mathrm{X}$-ray SLD values, which were calculated from the formula unit composition and mass densities for the various phases [21], are presented in Table I. From comparison with the experimental result, it is clear that the scattering length density profiles in Fig. 2 describe the chemical profiles of the respective nitride rather than the oxide. This is crucial because it shows that the thin-film capping layer functions correctly and prevents the formation of oxides or oxide-nitrogen nanocomposites. The rare-earth nitride (YSZ cap) layer thicknesses were found to be 41.8 (18.9), 15 (31), and 30.2 (22) $\mathrm{nm}$ for DyN, HoN, and ErN, respectively. From the fitted x-ray depth profile, a sharp interface is evident for all three films at the rare-earth nitride-sapphire boundary, corresponding to low substrate roughness $(0.5-0.6 \mathrm{~nm})$. The roughness of the rare-earth nitride to the YSZ cap interface was found to be 4.8, 0.8 , and $1.0 \mathrm{~nm}$ for DyN, HoN, and ErN, respectively, with a significantly rougher interface apparent for the case of DyN.

Figure 3 displays the polarized neutron reflectometry patterns obtained at $5 \mathrm{~K}$ for the DyN, HoN, and ErN thin films, along with the fits to the data using the nuclear and magnetic profile depicted in the inset. The layer thicknesses and roughness profiles were adopted from the x-ray reflectometry fits, and the scattering length densities were fitted starting from the same number densities as for the x-ray calculation, in combination with the neutron-scattering cross sections corresponding to the natural abundance of rare-earth isotopes. The determined nuclear SLDs match well with the theoretical nitride values recorded in Table I. The small differences between experiment and theory lie at the level of uncertainty and, in any case, could be expected for small 1-3\% shifts in density of the polycrystalline materials or $\approx 0.2 \%$ deviations from natural abundance of certain rare-earth isotopes. Clearly the shifts are far smaller than would occur if oxide phases were present. (a)

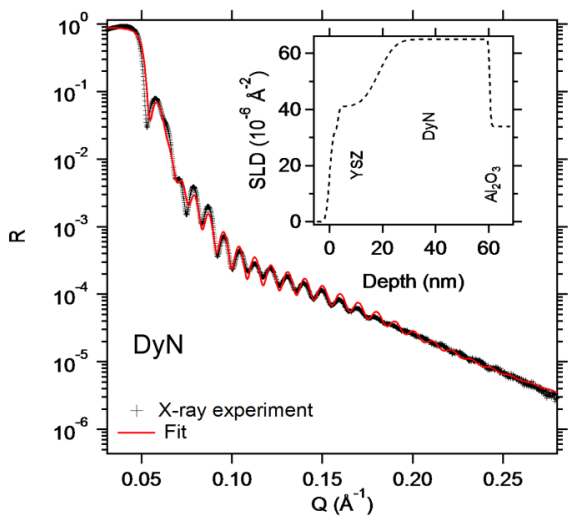

(b)

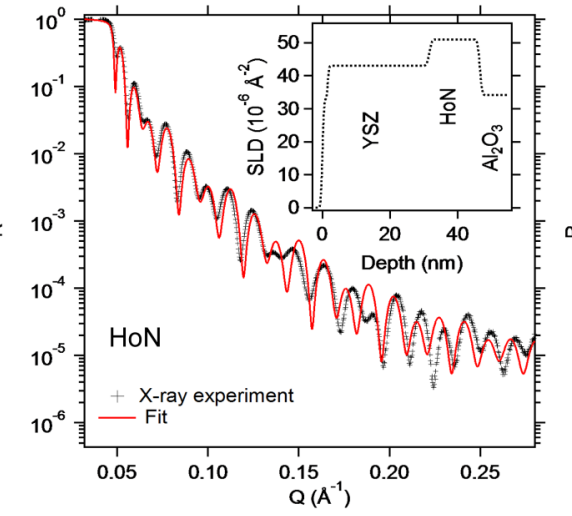

(c)

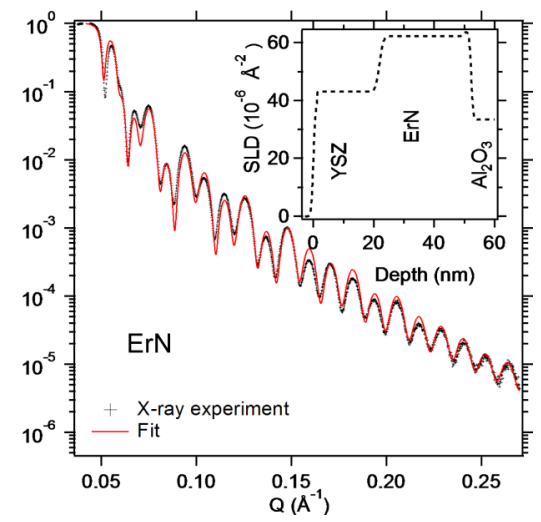

FIG. 2. (Color online) X-ray reflectometry patterns and fits to (a) DyN, (b) HoN, and (c) ErN thin films capped with YSZ. The black points are the data. The solid red line is a fit to the data. Inset: Best-fit scattering length density model. 
TABLE I. Material properties and x-ray and neutron nuclear scattering length densities. The mass densities and number densities $N$ (formula units per $\mathrm{m}^{3}$ ) have been taken from the literature values in [10,24-26]. The theoretical SLD values have been calculated assuming the latter number densities along with the natural abundances of the isotopes and the tabulated neutron and x-ray form factors for those isotopes [21]. The experimental SLD values correspond to the fitting results, where applicable.

\begin{tabular}{|c|c|c|c|c|c|c|}
\hline \multirow[b]{2}{*}{ Compound } & \multirow[b]{2}{*}{ Mass density $\left(\mathrm{g} / \mathrm{cm}^{3}\right)$} & \multirow[b]{2}{*}{$N\left(10^{28} \mathrm{~m}^{-3}\right)$} & \multicolumn{2}{|c|}{ X-ray $\operatorname{SLD}\left(10^{-6} \AA^{-2}\right)$} & \multicolumn{2}{|c|}{ Neutron nuclear SLD $\left(10^{-6} \AA^{-2}\right.$} \\
\hline & & & Theory & Experimental fit & Theory & Experimental fit \\
\hline DyN & 11.2 & 3.39 & 68.2 & 66.0 & 8.9 & 9.0 \\
\hline $\mathrm{HoN}$ & 10.3 & 3.47 & 53.0 & 51.0 & 6.0 & 6.2 \\
\hline $\mathrm{ErN}$ & 10.7 & 3.53 & 65.0 & 63.5 & 6.0 & 6.0 \\
\hline YSZ & 5.30 & 2.60 & 44.0 & 43.0 & 5.2 & 5.4 \\
\hline $\mathrm{Al}_{2} \mathrm{O}_{3}$ & 3.98 & 2.60 & 34.1 & 34.1 & 5.7 & 5.7 \\
\hline $\mathrm{Y}_{2} \mathrm{O}_{3}$ & 5.03 & 1.34 & 38.4 & & 4.4 & \\
\hline $\mathrm{Zr}_{2} \mathrm{O}_{3}$ & 5.68 & 2.85 & 43.4 & & 4.7 & \\
\hline $\mathrm{Dy}_{2} \mathrm{O}_{3}$ & 8.20 & 1.33 & 49.2 & & 6.8 & \\
\hline $\mathrm{Ho}_{2} \mathrm{O}_{3}$ & 8.40 & 1.34 & 44.9 & & 4.5 & \\
\hline $\mathrm{Er}_{2} \mathrm{O}_{3}$ & 8.60 & 1.36 & 54.0 & & 4.4 & \\
\hline
\end{tabular}

The fact that the chemistry of the films is extremely close to the ideal nitride is crucial because it supports the validity of the magnetic measurements presented in subsequent sections. The magnetic scattering length density for each layer was fitted as a free parameter while fixing all other structural parameters.

The magnetic moments, calculated by converting the fitted magnetic SLD into $\mu_{B}$ per formula unit [21], were determined at $5 \mathrm{~K}$ for the nitride and are recorded in Table II. The values agree well with previous magnetometry data for DyN and ErN thin films at the same field which is near saturation $[7,16]$; however, they are far lower than the theoretical predictions based on Hund's rules and density-functional theory [2]. Although our results agree well with the modern thin-film studies for ErN and DyN, the thin-film data do not match past bulk data. There are no pre-existing magnetic data for HoN thin films; however, it is clear that the magnetic moment found for HoN in the current study fits the trend established for DyN and ErN. Figure 4 compares our calculated moments per formula unit with the experimental and theoretical values reported in literature [7], including those predicted by DFT calculations based on local spin-density approximation with an additional
Hubbard energy (LSDA $+U)$ [2]. There is a wide scatter of experimental values in the literature for the bulk nitrides, whereas the modern thin-film studies agree quite closely $[7,16]$.

Both the chemical and structural measurements seem to exclude long-range oxidation throughout the rare-earth nitride layers; however, a valid question is whether any interfacial effects occur. For example, interface-localized oxidation or magnetic dead layers brought about by the close proximity to aluminium oxide or yttria could subtly alter the overall magnetic moment of the thin film. Nevertheless, the sub-nm resolution of neutron reflectivity allows us to rule out significant magnetic dead layers since deviation from the ideal values of DyN, HoN, and ErN no longer fits the data adequately. A method to check for interface effects, or inhomogeneity, is to subdivide the REN model into different regions and check whether the fit can be improved using different parameters for the sublayers. To demonstrate this point, we create a model where, due to the sensitivity to oxidation, the HoN within 1-2 nm of the YSZ oxide cap became partially oxidized or less magnetic. Figure 5(a) shows the case where a 2-nm-thick nonmagnetic interfacial layer is introduced to the HoN model just below the rare-earth-cap interface, with the consequence
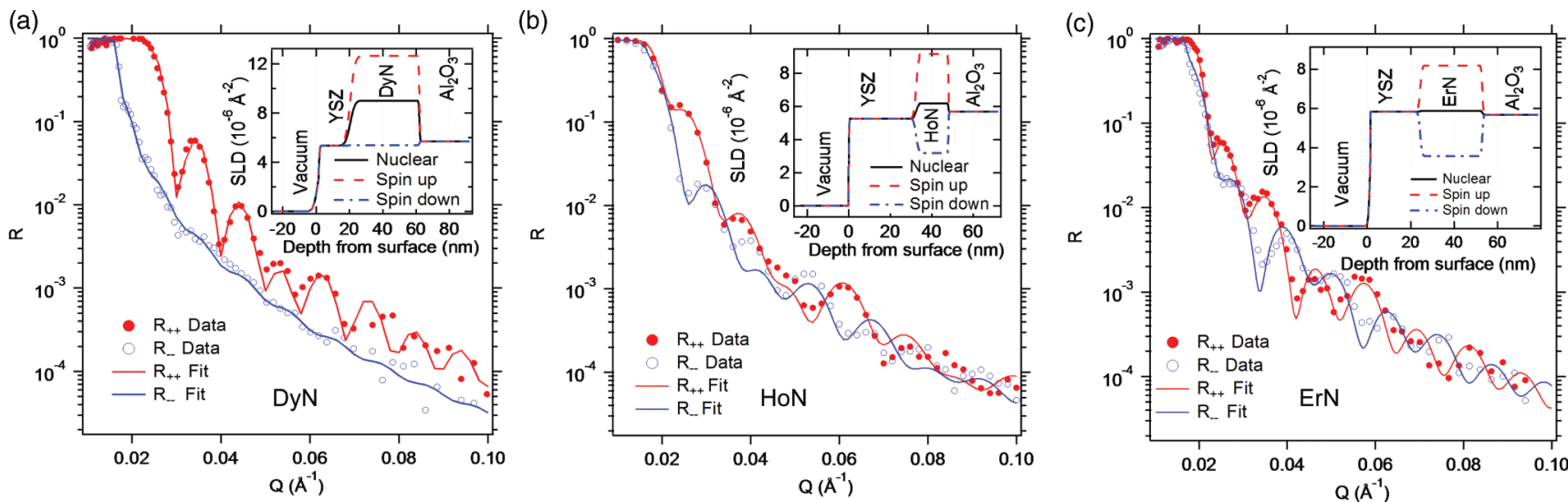

FIG. 3. (Color online) Polarized neutron reflectometry data and fit for (a) DyN, (b) HoN, and (c) ErN thin films capped with YSZ. The points are the data in the two spin channels. The solid lines are the fits to data. Inset: Best-fit scattering length density model. The field applied was $1 \mathrm{~T}$ for DyN and ErN and $3 \mathrm{~T}$ for HoN. 
TABLE II. Magnetic moment per rare earth at $5 \mathrm{~K}$ recorded by PNR at $1 \mathrm{~T}$ for DyN and ErN and $3 \mathrm{~T}$ for HoN.

\begin{tabular}{lcc}
\hline \hline Rare-earth nitride & $\mu_{B}$ per formula unit & $\mathrm{emu} / \mathrm{cm}^{3}$ \\
\hline DyN & 3.95 & 1300 \\
HoN & 3.2 & 960 \\
ErN & 2.3 & 800 \\
\hline \hline
\end{tabular}

that the calculated reflectivity no longer fits the experimental data. To better quantify this effect, it is helpful to consider the residual error $r_{U}$ between the model and experimental reflectivity patterns for different combinations of nuclear scattering length density and magnetic moment in regions of the rare-earth nitride film using the formula

$$
r_{U}=\sum\left(\frac{R_{\text {exp }}(Q)-R_{\text {calc }}(Q)}{R_{\text {exp }}(Q)+R_{\text {calc }}(Q)}\right)^{2} .
$$

The summation is performed from the residuals for all points in both the spin-up and spin-down channels. Figure 5(b) is the residual error, where the two parameters of neutron nuclear SLD (chemical composition) and magnetic moment per HoN formula unit have been varied for the whole HoN thin film. The position of the white cross corresponds to the best-fit parameters recorded in Tables I and II. Figure 5(c) presents the calculations performed where only a 2-nm interface region near the capping layer was modified, with a variety of nuclear and magnetic scattering length density combinations. The well-localized minimum is the same as the one found for the bulk film properties of the HoN layer, showing that decreasing, or increasing, the calculated interface moment led to an increase in fit error. Similar features were seen for DyN and ErN and for different combinations of sublayer

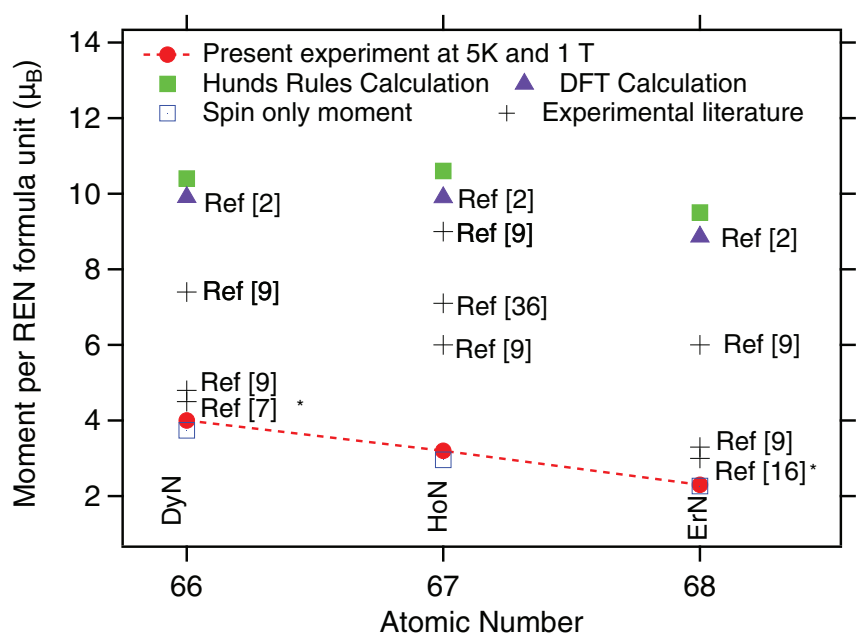

FIG. 4. (Color online) Ordered magnetic moment per rare-earth nitride formula unit compared with Hund's rules, DFT calculation, past literature, and the current experiment. Bulk literature values were adopted from the ordered state using $25-\mathrm{kOe} 5-\mathrm{K}$ neutron-diffraction data in [9]. The asterisk indicates other thin-film studies in $[18,19]$ using the 5-K and 1-T values to be directly comparable with the present study. The spin-only Hund's rule calculation moment assumes that the angular momentum is quenched so that $J=S$. thicknesses. Collectively this analysis indicates that the REN films are chemically and magnetically homogeneous, and the layer mixing at the interface does not introduce any nontrivial magnetic or chemical effects that can be detected. A second question is whether the samples are truly in the ferromagnetic state at $5 \mathrm{~K}$. Previously a very low Curie temperature was reported for $\operatorname{ErN}\left(\mathrm{T}_{c}=6.3 \mathrm{~K}\right)$ [16], whereas the Curie temperature of DyN was found to be higher $\left(\mathrm{T}_{c}=43 \mathrm{~K}\right)$ [7]. Irreversible or thermoremnant magnetization is a clear signature of long-range ferromagnetism. To check for this feature in DyN, one can compare the field-cooled and zero-field-cooled neutron data at $5 \mathrm{~K}$, measured at low magnetic fields, shown in Fig. 6.

The strong spin asymmetry for the case of DyN (the difference between $\mathrm{R}_{++}$and $\mathrm{R}_{--}$) for the field-cooled state is a direct product of the formation of thermoremnant magnetization concurrent with a stable ferromagnetic component and a sufficiently large coercive field. In contrast, for HoN and ErN, the field-cooled and zero-field-cooled data were found to be essentially identical at $5 \mathrm{~K}$ and showed no detectable spin asymmetry at low fields, implying a low coercive field. The results agree with previously published magnetometry for ErN and DyN thin films [7,16] and indicate a similarity between HoN and ErN. It was suggested that a noncollinear spin structure would explain the lowered net moment in ErN [16]. If a coherent helical or spiral arrangement of magnetic moments were present in any of the films, this may result in a net transverse magnetic component as a function of depth which would be detectable in the neutron spin-flip signal, as shown in polarized neutron reflectometry (PNR) experiments of other rare-earth materials [27-30]. However, no spin-flip signal was detected in the polarized neutron experiment for DyN, HoN, or ErN above background. This, however, does not rule out the possible presence of noncollinear ferromagnetic spiral structures, because the random anisotropy directions for the crystallites of a polycrystalline film will potentially lead to equal populations of different chiralities giving a net cancellation of the transverse moment [29]. Figure 7 shows the magnetic hysteresis loops of HoN measured by superconducting quantum interference device (SQUID) magnetometry at low temperatures for a 260-nm-thick film, where the density and thickness found from $\mathrm{x}$-ray reflectometry fits are used to estimate the magnetization in units of Bohr magnetons per HoN. The data have been corrected for the diamagnetic contribution from the substrate and holder by subtracting the relevant data at $350 \mathrm{~K}$. It is clear that the ratio of the remanence to saturation is extremely small in HoN, although it exhibits a clear coercive field $(\approx 0.01 \mathrm{~T})$. While low, the temperature-dependent coercive field at $5 \mathrm{~K}$ is larger than the zero-point error of the SQUID (0.0001 T) and coercive fields for soft ferromagnets like $\mathrm{Ni}_{80} \mathrm{Fe}_{20}(0.0005 \mathrm{~T})$ studied before and therefore is unlikely to be an artifact. The coercive field cannot be caused by pure paramagnetism and provides an unambiguous indication of a degree of stable magnetic order with a ferromagnetic component. It is also true that many unconventional ferromagnets such as canted antiferromagnets or dilute magnetic semiconductors share a similar anhysteretic character in their hysteresis loops [31]. The magnetization at $5 \mathrm{~K}$ is almost saturated to a value of $\approx 3 \mu_{B}$ per HoN by the application of a 3-T field; however, there is some linear increase 
(a)

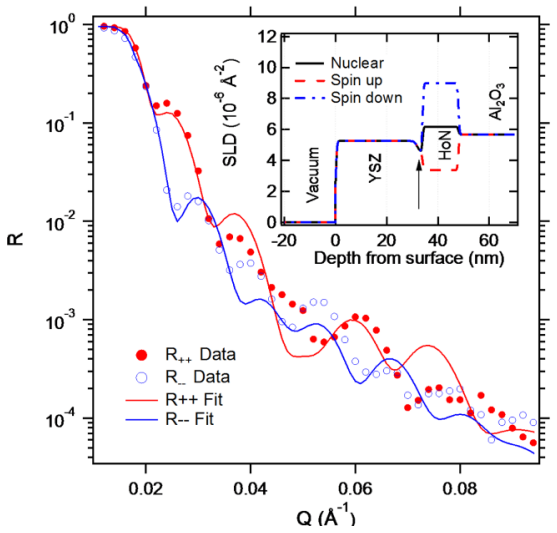

(b)

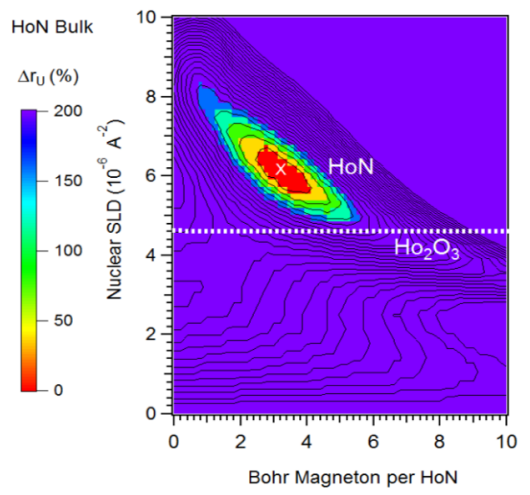

(c)

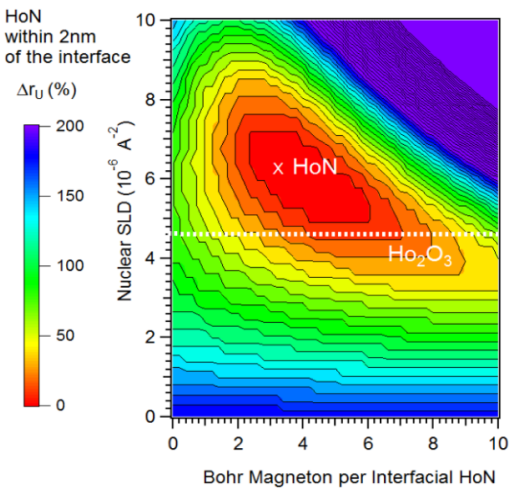

FIG. 5. (Color online) (a) An example of a poor fit resulting from a 2-nm interface with zero magnetic moment showing that, if they were present, oxidation and magnetic dead layers would be detectable by polarized neutron reflectometry. The arrow points out the 2-nm interface region. Contour plots show the residuum dependency on magnetic moment and nuclear scattering length density for the (b) bulk film properties and (c) 2-nm interface of $\mathrm{HoN}$. The white crosses indicate the minima while the white lines indicate the SLD for $\mathrm{Ho}_{2} \mathrm{O}_{3}$ for comparison.

in moment even up until the maximum field applicable in the MPMS. These features are similar to the case for ErN [16] and are qualitatively consistent with low exchange interactions for spins in a relatively strong crystal-field environment which result in similar general features for the other monopnictides [32]. Another possibility, known from Ho metallic films [27,29,33-35], is that thickness dependent effects alter the magnetic structure; however, we attempted to exclude that situation here by studying two HoN films with different thicknesses. Figure 8 shows the dependency of the magnetization of HoN for a 260- and 15-nm-thick film measured by SQUID magnetometry and PNR. The PNR data at $3 \mathrm{~T}$ for the 15-nm-thick film are within $20 \%$ of the $260-\mathrm{nm}$ film, which may be explained from uncertainties from the magnetometry pertaining to the diamagnetic correction and sample cross section. As a second argument against a thickness dependent effect, the neutron data presented here for the $\approx 20$-nm DyN and ErN thin films also match quite well with

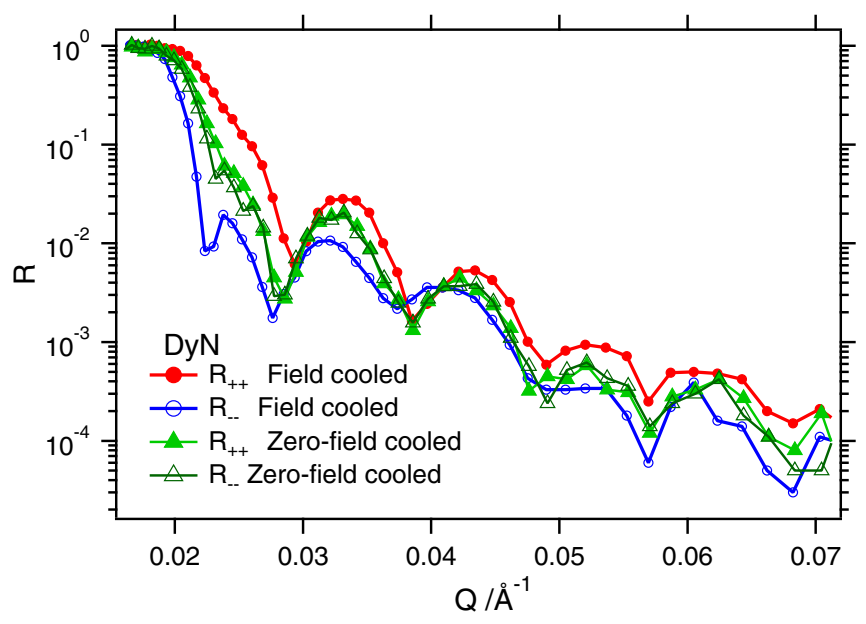

FIG. 6. (Color online) Reflectivities for DyN at $5 \mathrm{~K}$ in a measurement field of $0.01 \mathrm{~T}$ after either field cooling from $70 \mathrm{~K}$ in a field of $1 \mathrm{~T}$ or zero field. The measurement is near the minimal field required to keep the neutrons polarized. the magnetometry on thicker films (200-nm thick) previously published $[7,16]$. This suggests that thickness effects in the heavy rare-earth nitride films do not provide a convincing explanation for the reduction of the moment by a factor of 3.5 from the theoretical $\mathrm{Ho}^{3+}$ ion $\left(10.6 \mu_{B}\right)$. Collapse of magnetic moment from the ionic expectation has been noted generally in the rare-earth monopnictides $[9,36]$. For HoN in Fig. 8, an increase in susceptibility is observed near the reported Curie bulk temperature (18 K) [9]; however, no bifurcation is apparent in the field-cooled and zero-field-cooled data. It is challenging to accurately determine the Curie-Weiss temperature from the neutron reflectometry or magnetometry data because the inverse susceptibilities (see inset of Fig. 8) are nonlinear above $200 \mathrm{~K}$, leading to different effective paramagnetic moments ( $\approx 10 \mu_{B}<200 \mathrm{~K}$ and $\approx 7.5 \mu_{B}$ in the 200 - to 300 -K regime). The higher temperature transition suggests that other low-lying Ho crystal-field levels with different total angular momentum may exist at energies which can become thermally populated. In the absence of reliable bulk magnetization data and inelastic neutron data for HoN, we consider this an open question.

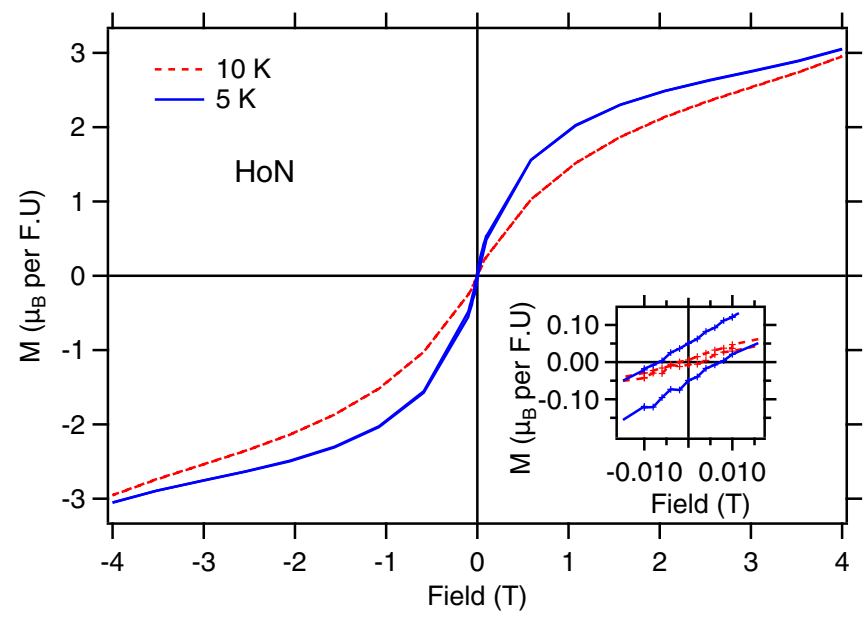

FIG. 7. (Color online) Magnetic hysteresis loops for $\mathrm{HoN}$ at 5 and $10 \mathrm{~K}$. Inset: Coercive field at $5 \mathrm{~K}$. 


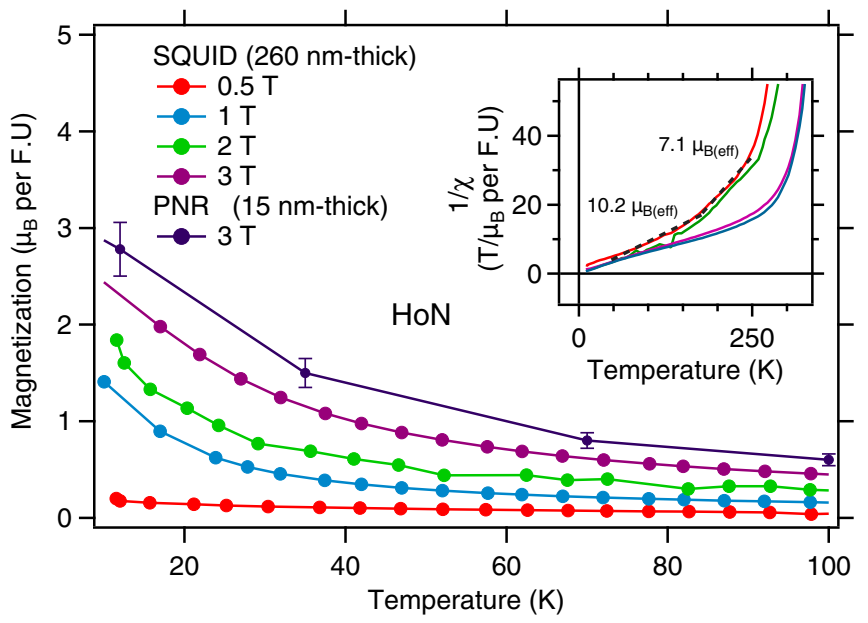

FIG. 8. (Color online) Magnetic moment as a function of temperature for HoN measured with SQUID magnetometry and compared with the neutron fitting results. Inset: Inverse susceptibility per formula unit of HoN.

\section{DISCUSSION AND CONCLUSIONS}

The magnetic properties of HoN thin films were reported, finding a large net magnetization of $960 \mathrm{emu} / \mathrm{cm}^{3}$ at $5 \mathrm{~K}$ and $1 \mathrm{~T}$ and small coercive field of $0.01 \mathrm{~T}$ in this semiconducting compound. The magnetic moment determined from polarized neutron reflectometry for ErN and DyN at $5 \mathrm{~K}$ and $1 \mathrm{~T}$ is in excellent agreement with the values derived from magnetometry on other films $[7,16]$. It is worth noting that, while the resulting magnetization in DyN, ErN, and HoN is comparable to that of ferromagnetic iron, the magnetic moment values reported in Table II for the RENs are lower than the moments predicted for the rare-earth $3+$ ion. The reduction of the net ferromagnetic moments in DyN, ErN, and $\mathrm{HoN}$ thin films appears to be an intrinsic feature which cannot be explained by detectable material imperfections or a finite-size effect. Our detailed analysis implies that the films are stoichiometric within 1-3\% and the measured magnetic moment reproduces the data from past thin films prepared under completely different conditions $[7,16]$. The use of two complementary reflectometry techniques proves that the chemical profiles of the rare-earth nitride films studied are essentially homogeneous and in agreement with bulk values, with no obvious formation of oxide layers at the interface between the cap and the nitride. The close quantitative agreement between our work and past studies of ErN and DyN $[7,16]$ is noteworthy since those studies used different deposition and capping methods, and so far there has been no attempt to control other variables like nitrogen vacancies, oxygen doping, or defect concentration. The apparent insensitivity to these parameters may be noteworthy given the current thinking that boundmagnetic polarons mediated via anion vacancies and carrier concentration may greatly enhance the exchange mechanism in $\operatorname{GdN}$ [37,38], and in the other RENs [39], in analogy to other ferromagnetic semiconductors. Therefore, our key finding is that the magnetic moment measured is not affected by unwanted chemical effects either because they are not present, or, if some small oxygen doping exists below the detection limit, it is not critically important to the magnetic properties of ErN, DyN, and HoN, in contrast to the case of GdN. This also implies that the reduced moment is intrinsic and related to a complex spin structure induced by crystal-field effects, as proposed for the intrinsic properties of the RE nitrides [32]. Strong crystal fields could also lead to partial quenching of the angular momentum, although according to an earlier theoretical model for $\mathrm{HoN}$, the saturation moment for $\mathrm{HoN}$ should only be reduced to be $9.4 \mu_{B}$ [40]. The underestimation by the existing DFT calculations implies additional electron correlations play an important role in the magnetism. Recently it was shown that a Ho $4 f-\mathrm{N} 2 p$ hybridization occurs for HoN films [17], evident in an altered band structure [2]. Such hybridization could promote the quenching of angular momentum or shielding of the $4 f$ moment. This aspect was not considered in past theoretical calculations $[17,40]$. The current work has established that thin-film growth and capping methods employed are suitable for stabilizing polycrystalline nitrides with reproducible properties. However, high-angle neutron-diffraction data and element-resolved circular $\mathrm{x}$-ray dichroism data would assist in determining the dominant physical mechanisms for the collapse of magnetic moment. The fabrication of epitaxial films with variable thickness for these types of studies is an ongoing challenge for us and other researchers. If the crystal-field model proposed by Trammel is the correct explanation for the reduced moment, it follows that there is also the possibility of a linear Dirac-cone type of spin-wave dispersion in these magnetically ordered semiconductors $[32,41]$.

\section{ACKNOWLEDGMENTS}

The research presented herein is made possible by a reflectometer jointly funded by Canada Foundation for Innovation, Ontario Innovation Trust, Ontario Research Fund, and the National Research Council Canada. The access to major research facilities program was supported by the Commonwealth of Australia under the International Science Linkages program. D.L.C. acknowledges the support of the Australian Institute Nuclear Science and Engineering. Figure 1 was visualized using the VESTA software [42].
[1] C.-G. Duan, R. F. Sabirianov, W. N. Mei, P. A. Dowben, S. S. Jaswal, and E. Y. Tsymbal, J. Phys.: Condens. Matter 19, 315220 (2007).

[2] P. Larson, W. R. L. Lambrecht, A. Chantis, and M. van Schilfgaarde, Phys. Rev. B 75, 045114 (2007).
[3] F. Leuenberger, A. Parge, W. Felsch, K. Fauth, and M. Hessler, Phys. Rev. B 72, 014427 (2005).

[4] F. Leuenberger, A. Parge, W. Felsch, F. Baudelet, C. Giorgetti, E. Dartyge, and F. Wilhelm, Phys. Rev. B 73, 214430 (2006). 
[5] A. R. H. Preston, B. J. Ruck, W. R. L. Lambrecht, L. F. J. Piper, J. E. Downes, K. E. Smith, and H. J. Trodahl, Appl. Phys. Lett 96, 032101 (2010).

[6] K. Senapati, T. Fix, M. E. Vickers, M. G. Blamire, and Z. H. Barber, J. Phys.: Condens. Matter 22, 302003 (2010).

[7] A. R. H. Preston, S. Granville, D. H. Housden, B. Ludbrook, B. J. Ruck, H. J. Trodahl, A. Bittar, G. V. M. Williams, J. E. Downes, A. DeMasi, Y. Zhang, K. E. Smith, and W. R. L. Lambrecht, Phys. Rev. B 76, 245120 (2007).

[8] F. Natali, B. Ruck, N. Plank, H. Trodahl, S. Granville, C. Meyer, and W. Lambrecht, Progress in Materials Science 58, 1316 (2013).

[9] H. R. Child, M. K. Wilkinson, J. W. Cable, W. C. Koehler, and E. Wollan, Phys. Rev. 131, 922 (1963).

[10] R. Brown and N. Clark, J. Inorg. Nucl. Chem. 36, 2507 (1974).

[11] K. Senapati, M. G. Blamire, and Z. H. Barber, Nat. Mat. 10, 849 (2011).

[12] H. Warring, B. J. Ruck, H. J. Trodahl, and F. Natali, Appl. Phys. Lett. 102, 132409 (2013).

[13] G. Busch, J. Appl. Phys. 38, 1386 (1967).

[14] R. J. Gambino and T. McGuire, J. Appl. Phys 41, 933 (1970).

[15] J. Kennedy, S. Granville, A. Markwitz, B. J. Ruck, and H. J. Trodahl, Nucl. Instrum. Method. B 266, 1558 (2008).

[16] C. Meyer, B. J. Ruck, A. R. H. Preston, S. Granville, G. V. M. Williams, and H. J. Trodahl, J. Magn. Magn. Mat. 322, 1973 (2010).

[17] J. D. Brown, J. E. Downes, C. J. McMahon, B. C. C. Cowie, A. Tadich, L. Thomsen, J. H. Guo, and P. A. Glans, Appl. Phys. Lett. 100, 072108 (2012).

[18] M. James, A. Nelson, S. A. Holt, T. Saerbeck, W. A. Hamilton, and F. Klose, Nucl. Instr. Meth. Phys. Res. A 632, 112 (2011).

[19] H. Fritzsche, Rev. Sci. Instrum. 76, 115104 (2005).

[20] T. Saerbeck, F. Klose, A. L. Brun, J. Fzi, A. Brule, A. Nelson, S. Holt, and M. James, Rev. Sci. Instrum. 83, 081301 (2012).

[21] M. Fitzsimmons and C. Majkrzak, in Modern Techniques for Characterizing Magnetic Materials, edited by Y. Zhu (Springer, New York, 2005), pp. 107-155.

[22] A. Nelson, Journ. Appl. Cryst. 39, 273 (2006).
[23] http://www-llb.cea.fr/prism/programs/simulreflec/simulreflec. $\mathrm{html}$.

[24] G. Busch, P. Junod, O. Vogt, and F. Hulliger, Phys. Lett. 6, 79 (1963).

[25] D. Lupascu, A. Bartos, K. P. Lieb, and M. Uhrmacher, Zeitschrift für Physik B Condensed Matter 93, 441 (1994).

[26] E. Maslen, V. Streltsov, and N. Ishizawa, Acta Crystallographica B 52, 414 (1996).

[27] G. P. Felcher, W. Lohstroh, H. Fritzsche, M. Münzenberg, H. Maletta, and W. Felsch, Appl. Phys. Lett. 72, 2894 (1998).

[28] W. Lohstroh, O. Schulte, F. Klose, M. Müzenberg, W. Felsch, H. Maletta, and H. Lauter, Physica B 234-236, 477 (1997).

[29] S. V. Grigoriev, Y. O. Chetverikov, D. Lott, and A. Schreyer, Phys. Rev. Lett. 100, 197203 (2008).

[30] H. Fritzsche, M. Saoudi, Z. Yamani, W. J. L. Buyers, R. A. Cowley, and R. C. C. Ward, Phys. Rev. B 77, 054423 (2008).

[31] J. M. Coey, New J. Phys. 12, 053025 (2010).

[32] G. T. Trammel, Phys. Rev. 131, 932 (1963).

[33] G. Felcher, G. Lander, T. Arai, S. Sinha, and F. Spedding, Phys. Rev. B 13, 3034 (1976).

[34] V. Leiner, D. Labergerie, R. Siebrecht, C. Sutter, and H. Zabel, Physica B 283, 167 (2000).

[35] J. Bowlan, C. N. van Dijk, A. Kirilyuk, A. Liang, S. Yin, T. Rasing, and W. A. de Heer, J. Appl. Phys. 107, 09B509 (2010).

[36] T. A. Yamamoto, T. Nakagawa, K. Sako, T. Arakawa, and H. Nitani, J. Alloy. Compounds 376, 17 (2004).

[37] F. Natali, B. J. Ruck, H. J. Trodahl, D. L. Binh, S. Vezian, B. Damilano, Y. Cordier, F. Semond, and C. Meyer, Phys. Rev. B. 87, 035202 (2013).

[38] N. O. V. Plank, F. Natali, J. Galipaud, J. H. Richter, M. Simpson, H. J. Trodahl, and B. J. Ruck, Appl. Phys. Lett. 98, 112503 (2011).

[39] B. J. Ruck, F. Natali, N. O. V. Plank, B. Le, M. Azeem, M. Alfheid, C. Meyer, and H. J. Trodahl, Physica B 407, 2954 (2012).

[40] Y. Ebina, J. Phys. Soc. Japan 18, 189 (1963).

[41] T. A. Kaplan, Phys. Rev. 124, 329 (1961).

[42] K. Momma and F. Izumi, J. Appl. Crystallogr. 44, 1272 (2011). 